\title{
Stabilization of Vertically Modulated Pendulum with Parametric Periodic Forces
}

\author{
Babar Ahmad* Sidra Khan*
}

\begin{abstract}
With the application of Kapitza method of averaging for arbitrary periodic force, a vertically modulated pendulum, with periodic linear forces is stabilized by minimizing its potential energy function. These periodic linear forces are selected in range [-1, 1], further the corresponding stability conditions are compared with that in case of harmonic modulation. Later, a parametric control is defined on some periodic piecewise linear forces, and the nontrivial position is stabilized under different conditions by just adjusting the parameter.
\end{abstract}

Keywords: Kapitza pendulum, fast oscillation, parametric control

\section{Introduction}

A simple pendulum that is suspended under the influence uniform gravitational field has versatile applications in Nonlinear Physics. The Mathematical Relationships and the differential equations associated with pendulum plays an important role in the theory of solutions, in the problem of super radiation, in quantum optics and the theory of Josephson effects in weak superconductivity [1]. A simple pendulum has only one stable point i.e. vertically downward position, while a vertically modulated pendulum with very high frequency, has upward position also stable. This concept was initialized by Stephenson in 1908.[2, 3, 4]. In 1951, Pjotr Kapitza explained experimentally such kind of extraordinary behavior of pendulum in detail, and corresponding experimental instrument is known as Kapitza Pendulum [5]. In 1960 Landau et al. examined the stability of this system driven by harmonic Force [6]. Later on, Ahmad and Borisenok replaced harmonics force with periodic kicking forces and modified Kapitza Method for arbitrary periodic forces [7]. Ahmad also examined the stability of the system excited by the symmetric forces with comparatively low frequency of fast Oscillations [8]. Later on, the behavior and the stability of a parametrically excited pendulum have been examined $[9,10]$. In 2013, Ahmad used parametric periodic linear forces for the horizontal modulated pendulum and discussed its stability by minimizing the potential energy function [11]. In this paper, the stability criterion for vertically modulated pendulum, driven by periodic piecewise linear forces will be discussed.

\section{Kapitza Method For Periodic Arbitrary Forces with Zero Mean}

Consider one dimensional motion of a particle of mass $\mathrm{m}$ in conservative system. If $\mathrm{U}$ is potential energy function, then its equation of motion is

$$
F(x)=\frac{-d U}{d x}
$$

In this case the system has only one stable point. If a periodic fast oscillating force with zero mean is introduced, The system may have more than one stable point. This fast oscillation means that if $\omega_{0}=\frac{2 \pi}{T_{0}}$ is the frequency due to $F_{1}$ and $\omega=\frac{2 \pi}{T}$ is the frequency due $F_{2}$ then $\omega>>\omega_{0}$. This fast oscillatory force has the Fourier expansion as

$$
F_{2}(x, t)=\sum_{k=0}^{\infty}\left[a_{k}(x) \cos (k w t)+b_{k}(x) \sin (k w t)\right]
$$

Here $a_{k}$ and $b_{k}$ are the Fourier coefficients. In Calculus, mean value of a function $f(t)$ is denoted by bar, if $T$ is the time period, then mean is defined as

$$
\bar{f}=\frac{1}{T} \int_{0}^{T} f(x, t) d t
$$

The Fourier coefficient $a_{0}$ is defined as

$$
a_{0}=\frac{2}{T} \int_{0}^{T} f_{2}(x, t) d t
$$

${ }^{*}$ COMSATS Institute of Information Technology Islamabad, Pakistan Corresponding Email: baber.sms@gmail.com 
From equation 3 and 4 , the mean value of a function is equivalent to Fourier coefficient $a_{0}$

$$
\bar{f} \cong a_{0}(x)
$$

The other Fourier coefficients are

$$
\begin{aligned}
& a_{k}=\frac{2}{T} \int_{0}^{T} f_{2}(x, t) \cos (k w t) d t \\
& b_{k}=\frac{2}{T} \int_{0}^{T} f_{2}(x, t) \sin (k w t) d t
\end{aligned}
$$

Ignoring friction, we can say that only two forces are acting on the system, hence its equation of motion is

$$
m \ddot{x}=F_{1}(x)+F_{2}(x, t)
$$

Due to these forces, two types of motion namely smooth and small oscillations are observed. So we represent the path of oscillations as the sum of smooth path $X(t)$ and small oscillation $\xi(t)$

$$
x(t)=X(t)=\xi(t)
$$

By averaging procedure, the effective potential energy function can be expressed as

$$
U_{\text {eff }}=U+\frac{1}{4 m w^{2}} \sum_{k=1}^{\infty} \frac{a_{k}^{2}+b_{k}^{2}}{k^{2}}
$$

For stability of the system, we have to minimize effective potential energy function given by 8

\section{The Pendulum Driven by Harmonic Force}

Consider a pendulum whose pivot point is forced to vibrate vertically (see Figure 1), under the influence of the harmonic force. The harmonic force is given as

$$
f(t)=\sin (w t) \text { if } 0 \leq t \leq T
$$

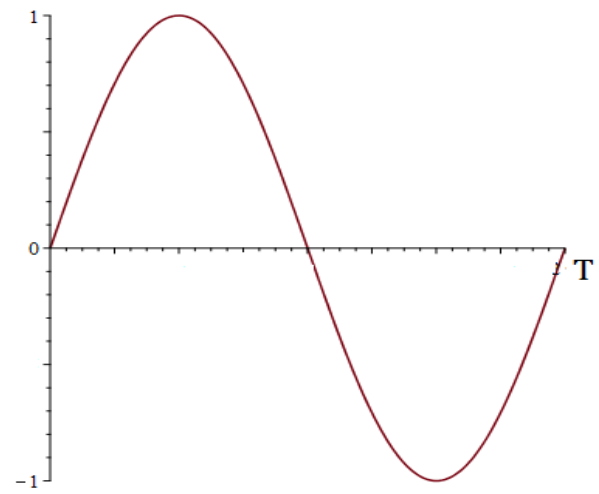

Figure 1: Kaptiza Pendulum with Vertical Oscillation

and shown in Figure 2

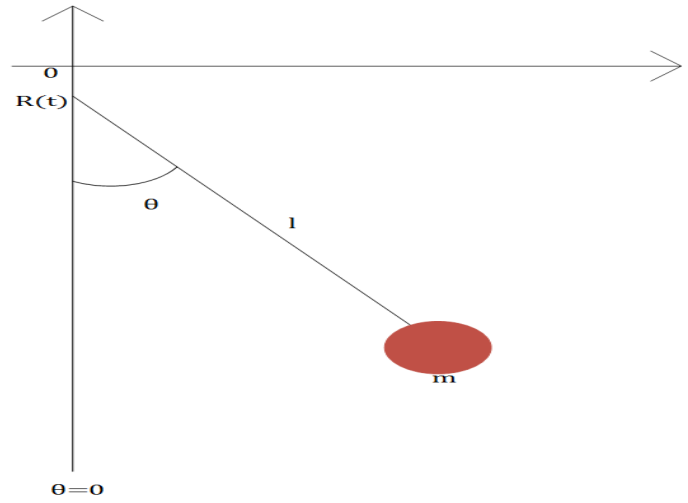

Figure 2: sin type force

And the force acting on the pendulum is

$$
f_{2}(\phi, t)=m w^{2} \sin \phi \times f(t)
$$

Its Fourier coefficient is $a_{0}=0$ indicates that its mean value is zero. By using 6, the other Fourier coefficients are:

$$
\begin{array}{r}
a_{k}=0 \\
b_{k}=m w^{2} \sin \phi
\end{array}
$$

so the effective potential energy is obtained by using 8

$$
U_{e f f}=m g l\left(-\cos \phi+\frac{w^{2}}{4 g l} \sin ^{2} \phi\right)
$$

The following results are obtained after minimizing equation 12

- The downward position $\phi=0$, is always stable.

- Vertically upward position $\phi=\pi$ is stable if $w^{2}>2 g l$.

- The position $\phi=\arccos \left(-\frac{2 g l}{w^{2}}\right)$ is unstable.

\section{Vertically Modulated Pendulum Driven by Periodic Linear Forces}

Now, replacing the harmonic force with some periodic piece-wise linear forces within the range of harmonic forces, Our aim is to stabilize the pendulum at $\phi=\pi$ with low frequency as compared to harmonic force. These periodic linear forces are T-periodical: $S(t+T) \equiv S(t)$. These forces are considered as following.

$$
f_{2}(\phi, t)=m w^{2} \sin \phi \times S(t)
$$

Inclined Type Force: First of all consider an inclined type force: $E(t)=E(t+T)$, given by equation 14 and illustrated in Figure 3

$$
E(t)=-\frac{2}{T} t+1 \text { if } 0 \leq t \leq T
$$




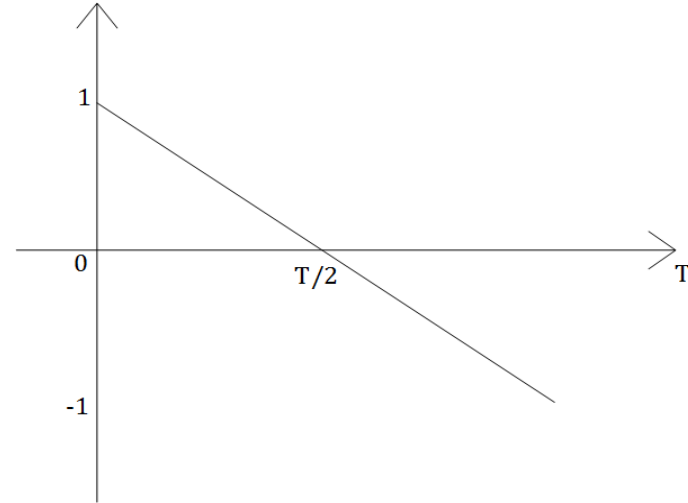

Figure 3: Inclined Type Force

The force acting on the particle is

$$
f(t)=m w^{2} \sin \phi \times E(t)
$$

The Fourier coefficient $a_{0}=0$, indicates that $\bar{E}=0$, the other Fourier coefficients are

$$
\begin{array}{r}
a_{k}=0 \\
b_{k}=m w^{2} \sin \phi\left(\frac{2}{k \pi}\right)
\end{array}
$$

So its potential energy function will be

$$
\begin{array}{r}
U_{e f f}=U+m w^{2} \sin ^{2} \phi \times \frac{1}{\pi^{2}} \sum_{k=0}^{\infty}\left(\frac{1}{k^{4}}\right) \\
=U+0.1097 m w^{2} \\
\sin ^{2} \phi
\end{array}
$$

Where $\phi=0, \pi$ and $\arccos \left(-\frac{4.5579 g l}{w^{2}}\right)$ are the extremum of 17 . After minimizing $17, w^{2}$ we have following results.

- The downward position $\phi=0$, is always stable.

- Vertically upward position $\phi=\pi$ is stable if $w^{2}>4.5579 g l$.

- The point $\phi=\arccos \left(-\frac{4.5779 g l}{w^{2}}\right)$ is unstable.

Quadratic Type force: Next, consider a quadratic type force: $Q(t)=Q(t+T)$ (shown is Figure 4), given by equation 18

$$
Q(t)= \begin{cases}1, & 0 \leq t<\frac{3 T}{8} \\ \frac{8}{T}\left(\frac{T}{2}-t\right), & \frac{3 T}{8} \leq t<\frac{5 T}{8} \\ -1, & \frac{5 T}{8} \leq t<t\end{cases}
$$

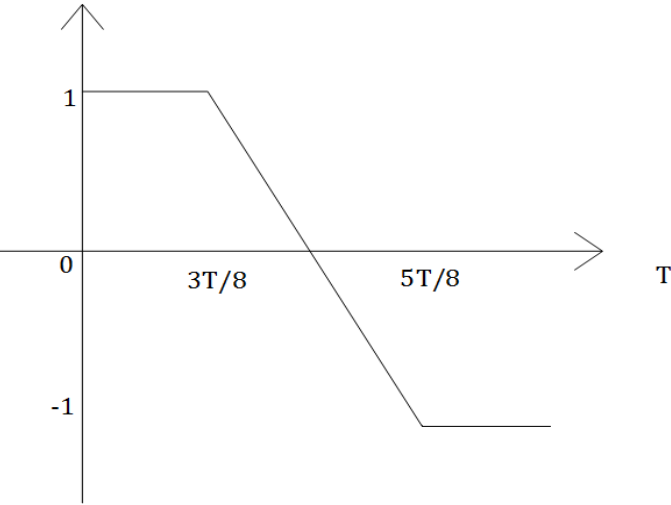

Figure 4: Quadratic type force

The force acting upon the particle is

$$
f(t)=m w^{2} \sin \phi \times Q(t)
$$

The fast oscillating force in Fourier expansion is given

$$
Q(t)=m w^{2} \sin \phi \sum_{k=1}^{\infty}\left(\frac{2}{k \pi}+\frac{8}{\pi^{2} k^{2}} \sin k \frac{\pi}{4}\right) \sin k(w t)
$$

With the following Fourier coefficients

$$
\begin{array}{r}
a_{k}=0 \\
b_{k}=m w^{2} \sin \phi \sum_{k=1}^{\infty}\left(\frac{2}{k \pi}+\frac{8}{\pi^{2} k^{2}} \sin k \frac{\pi}{4}\right)
\end{array}
$$

So the effective potential energy function will be

$$
\begin{array}{r}
U_{e f f}=U+m w^{2} \sin ^{2} \phi \times \frac{1}{4} \sum_{k=1}^{\infty} \frac{1}{k^{2}}\left(\frac{2}{k \pi}+\frac{8}{\pi^{2} k^{2}} \sin k \frac{\pi}{4}\right)^{2} \\
=U+0.3856 m w^{2} \\
\sin ^{2} \phi
\end{array}
$$

Where $\phi=0, \pi$ and $\arccos \left(-\frac{1.2967 g l}{w^{2}}\right)$ are the extremum of above system. With 21 , the stability of the system is given as

- The point $\phi=0$, is always stable.

- The point $\phi=\pi$ is stable if $w^{2}>1.2967 \mathrm{gl}$.

- The nontrivial position $\phi=\arccos \left(-\frac{1.2967 g l}{w^{2}}\right)$ is unstable.

So, it is observed that, the position $\phi=\pi$ is stabilized at lower frequency as compared to harmonic force.

Rectangular Type Force: Let's introduce the rectangular type force $R(t)=R(t+T)$, and the function $R(t)$ is T-periodic, given in 22, illustrated in Figure 5

$$
R(t)= \begin{cases}1, & 0 \leq t<\frac{T}{2} \\ -1, & \frac{T}{2} \leq t<T\end{cases}
$$




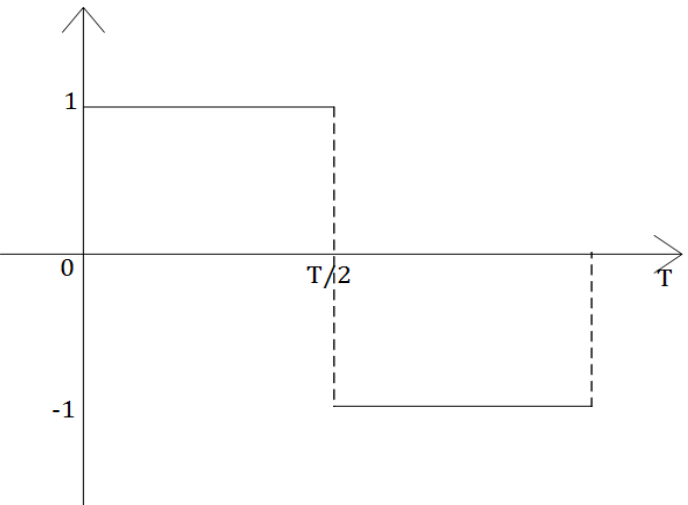

Figure 5: Rectangular type force

For vertical modulation, the force acting upon the particle is

$$
f(t)=m w^{2} \sin \phi \times R(t)
$$

The Fourier coefficient $a_{0}=0$, shows that $\bar{f}=0$, the other coefficients are

$$
\begin{array}{r}
a_{k}=0 \\
b_{k}=m w^{2} \sin \phi\left(\frac{4}{2 k-1}\right)
\end{array}
$$

Using above coefficients, the Fourier expansion is

$$
R(t)=m w^{2} \sin \phi \frac{4}{\pi} \sum_{k=1}^{\infty} \frac{1}{(2 k-1)} \sin (2 k-1) w t
$$

The effective potential energy is

$$
\begin{array}{r}
U_{e f f}=U+m w^{2} \sin ^{2} \phi \times \frac{1}{4}\left(\frac{16}{\pi^{2}}\right)^{2} \sum_{k=1}^{\infty} \frac{1}{(2 k-1)^{2}} \\
=U+0.4112 m w^{2} \\
\sin ^{2} \phi
\end{array}
$$

With the extremum at $\phi=0, \pi$ and $\arccos \left(-\frac{1.2159 g l}{w^{2}}\right)$. After minimizing 24, we have following results

- The point $\phi=0$, is always stable.

- The point $\phi=\pi$ is stable if $w^{2}>1.2159 \mathrm{gl}$.

- The nontrivial position $\phi=\arccos \left(-\frac{1.2159 g l}{w^{2}}\right)$ is unstable.

From the above examples, it is noticed that, at position $\phi=\pi$, the system is stabilized at lower frequency as compared to previous cases. The above results are summarized in Table 4.. It is also observed that, at nontrivial position, as area under the curve increases, the frequency of oscillation decreases. Harmonic and inclined type force has minimum area so they have maximum frequency as compared to rectangular type force.

\section{Parametric Control}

Next, a parametric control is defined on quadratic type force, to control the non-trivial position $\phi=\pi$. This force is also T-periodic, $Q_{\epsilon}(t+T)=Q_{\epsilon}(t)$. The control is defined for $0<\epsilon<1$. This $\epsilon$-parametric force is defined as

$$
Q_{\epsilon}(t)= \begin{cases}1 & 0 \leq t<\frac{1-\epsilon T}{2} \\ \frac{1}{\epsilon}\left(-\frac{T}{2} t+1\right) & \frac{1-\epsilon T}{2} \leq t<\frac{1+\epsilon T}{2} \\ -1 & \frac{1-\epsilon T}{2} \leq t<T\end{cases}
$$

and illustrated in Figure 6

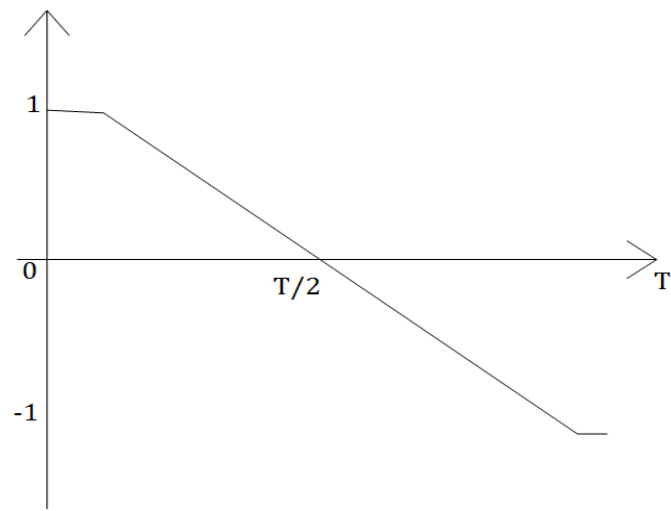

Figure 6: parametric quadratic type force

For vertical modulation the force acting upon the particle is

$$
f_{2}(\phi, t)=m w^{2} \sin \phi \times Q_{\epsilon}(t)
$$

From 25, the other Fourier coefficients are

$$
\begin{array}{r}
a_{k}=0 \\
b_{k}=m w^{2} \sin \phi\left(\frac{2}{k \pi}+\frac{8}{\epsilon \pi^{2} k^{2}} \sin k \frac{\pi}{4}\right)
\end{array}
$$

Fourier expansion of oscillating force is

$f_{2}(\phi, t)=m w^{2} \sin \phi \sum_{k=1}^{\infty}\left(\frac{2}{k \pi}+\frac{8}{\left(\epsilon \pi^{2} k^{2}\right)} \sin k \frac{\pi}{4}\right) \sin k w t \phi$

So, the effective potential energy will be

$$
\begin{aligned}
U_{e f f}=U+m w^{2} \sin ^{2} \phi \times & \frac{1}{4 \pi^{2}} \sum_{k=1}^{\infty} \frac{4}{k^{4}}\left(1+\frac{1}{\epsilon k \pi} \sin \epsilon k \pi\right)^{2} \\
& =-m g l \cos \phi+m w^{2} \sin ^{2} \phi . A
\end{aligned}
$$

and

$$
A=\frac{1}{\pi^{2}} \sum_{k=1}^{\infty} \frac{1}{k^{4}}\left(1+\frac{1}{\epsilon k \pi} \sin \epsilon k \pi\right)
$$

The effective potential energy 29 has extremum at $\phi=0, \pi, \arccos \left(-\frac{0.5 g l}{w^{2} \cdot A}\right)$.

After minimizing 29, we have the following results

- The system is stable at point $\phi=0$. - If $w^{2}>\frac{0.5 g l}{w^{2} . A}$, then the system will be stable at
$\phi=\pi$. 
Table 1: Stability Comparison of different linear forces with harmonic force

\begin{tabular}{|l|c|l|c|c|}
\hline External Force & Position & Stability & Position & $\begin{array}{c}\text { Stability } \\
\text { Condition }\end{array}$ \\
\hline sin & 0 & always & $\pi$ & $w^{2}>2 g l$ \\
\hline Inclined & 0 & always & $\pi$ & $w^{2}>4.5579 g l$ \\
\hline Quadratic & 0 & always & $\pi$ & $w^{2}>1.2969 g l$ \\
\hline Rectangular & 0 & always & $\pi$ & $w^{2}>1.2159 g l$ \\
\hline
\end{tabular}

Table 2: Stability condition of $\epsilon$-parametric force at $\phi=\pi$

\begin{tabular}{|c|c|c|}
\hline $0<\epsilon<1$ & Sum A & Stability Condition \\
\hline 0.9 & 0.1320 & $w^{2}>3.7879 g l$ \\
\hline 0.8 & 0.1607 & $w^{2}>3.1114 g l$ \\
\hline 0.7 & 0.1956 & $w^{2}>2.5562 g l$ \\
\hline 0.6 & 0.2357 & $w^{2}>2.1213 g l$ \\
\hline 0.5 & 0.2793 & $w^{2}>1.7902 g l$ \\
\hline 0.4 & 0.3239 & $w^{2}>1.5437 g l$ \\
\hline 0.3 & 0.3664 & $w^{2}>1.3647 g l$ \\
\hline 0.2 & 0.4029 & $w^{2}>1.2400 g l$ \\
\hline 0.1 & 0.4287 & $w^{2}>1.1663 g l$ \\
\hline
\end{tabular}

- The nontrivial position $\phi=\arccos \left(-\frac{0.5 g l}{w^{2} . A}\right)$ is unstable.

The stability of the system for different values of $\epsilon$ is summarized in Table 2 .

For $\epsilon=0.9$, the infinite sum $A=0.1320$, and the effective potential energy function is

$$
U_{e f f}=-m g l \cos \phi+0.132 m w^{2} \sin ^{2} \phi
$$

At the position $\phi=\pi$, the system is stable if the condition $w^{2}>3.7879 \mathrm{gl}$ is satisfied, and this value is much greater than all previous results. Next for $\epsilon=0.8$, the infinite sum $A=0.1606$, and the point $\phi=\pi$ is stable is $w^{2}>3.1114 \mathrm{gl}$, which gives much better result. Similarly, For $\epsilon=0.1$, the system is stabilized at the same position with the condition $w^{2}>1.663 \mathrm{gl}$, and this result is better than all considered examples. From above discussed cases, it can be observed that, with the decrease in value of, infinite sum $\mathrm{A}$ is increased, thus stabilizing the system at relatively lower frequency at $\phi=\pi$.

It is also observed that, as $\epsilon \rightarrow 1$, the term $A \cong 0.1098$ and the system is stabilized at the position $\pi$ with the condition $w^{2}>3.4 .5537 \mathrm{gl}$, and this frequency is approximately equal to the inclined type force. Thus, the quadratic type force approaches to inclined type force as $\epsilon \rightarrow 1$, and the system is stabilized with much greater frequency and is not stable.

However, as $\epsilon \rightarrow 0$, the term $A \cong 0.4386$, and the position $\phi=\pi$ is stable if the condition $w^{2}>1.14 \mathrm{gl}$ is satisfied, and this frequency of oscillation is lower than rectangular type force. Observe the Table 2, the rectangular force fall between $\epsilon=0.2$ and $\epsilon=0.1$, and for the parametric force with $\epsilon=0.1$, the frequency of oscillation becomes lower than that in case of rectangular type force. Hence, by defining the parametric control better results are achieved.

\section{Conclusion}

Using Kaptiza method of averaging for arbitrary periodic forces, the vertically modulated pendulum excited by periodic linear forces is stabilized at $\phi=$ $\pi$ with the frequency $w$, that was found to be sufficiently less relative to the case of harmonic modulation. Moreover, the rectangular type force was found to be the best. The stability conditions at nontrivial position $\phi=\pi$ improves by defining a parametric control on some of the periodic piecewise linear forces. Hence, by adjusting the parameter, the system is stabilized with less oscillating frequency.

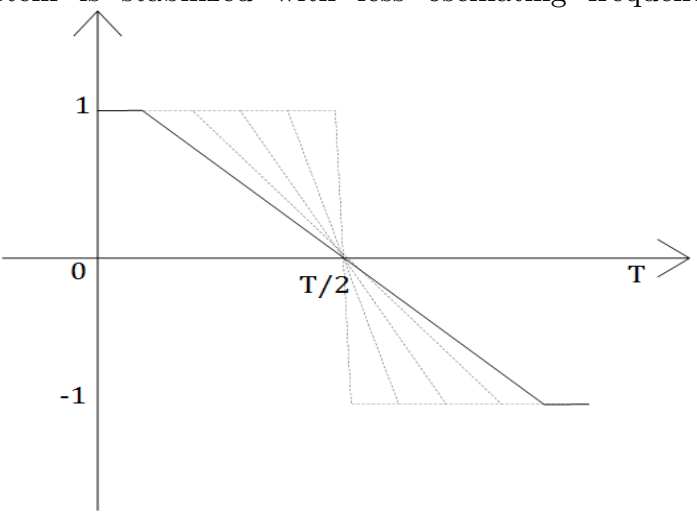

Figure 7: Quadratic type force for different values of $\epsilon$ 


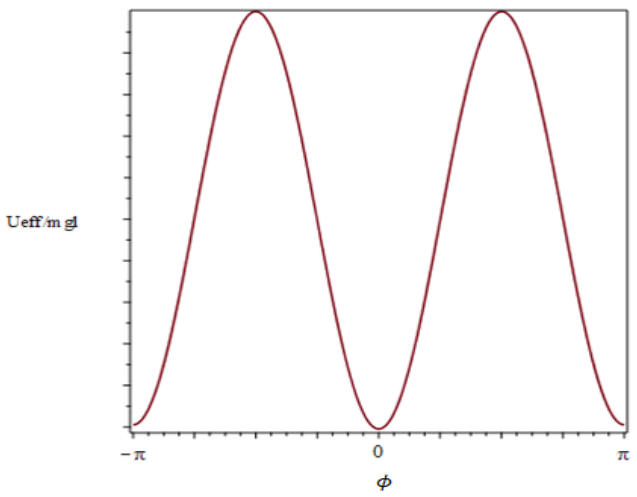

Figure 8: $U_{\text {eff }}$ is minimum at $\phi=\pi i f w^{2}>1.14 g l$

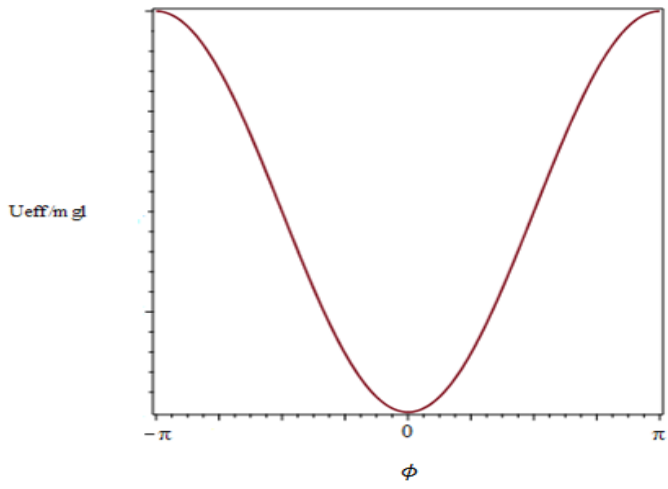

Figure 9: $U_{\text {eff }}$ is always minimum at $\phi=0$

\section{References}

[1] E. Butikov, "The rigid pendulum-an antique but evergreen physical model," European Journal of
Physics, pp. 424-441, 1999.

[2] A. Stephenson, "On induced stability," Philosophical Magzine, pp. 233-236, 1908.

[3] A. Stephenson, "On induced stability," philosophical Magzine, vol. 17, pp. 756-766, 1909.

[4] A. Stephenson, "On new type of dynamic stability," Memories and Proceeding of the Manchester Litrerary and Philosophical Magzine, pp. 110, 1908

[5] P. L. Kapitza, "Dynamic stability of pendulum with an oscillating point of suspension," Journal of Experimental and Theorectical Physics, pp. 588$597,1951$.

[6] E. M. Lifshitz, L. D. Landau and mecanics, Oxford, UK: Pergamon Press: butterworth, 2005.

[7] B. Ahamd and S. Borisenok, "Control of effective potential minima for Kapitza oscillator by periodical kicking pulses," Physics Letter A, pp. 701-707, 2009 .

[8] B. Ahmad, "Stabilization of Kapitza pendulum by symmetrical periodical forces," Nonlinear Dynamics, pp. 499-506, 2010.

[9] E. Butikov, "An improved criterian for Kapitza pendulum stability.," Journal of Physics A: Mathematical and Theoratical, vol. 44, 2011.

[10] E. Butikove, "Subharmonic resonances of the parametrically driven pendulum," Journal of Physics A: Mathematical. Gen, vol. 35, 2002.

[11] B. Ahmad, "Stabilization of driven pendulum with periodic linear forces," Nonlinear Dynamics, vol. 2013, 2013. 